\section{LA PREVENCIÓN DEL DELITO EN UNA VILLA DE EMERGENCIA EN BUENOS AIRES (ARGENTINA), INSERCIÓN Y PARTICIPACIÓN, ANÁLISIS DE LOS SUPUESTOS DE COMUNIDAD EN LAS POLÍTICAS DE PREVENCIÓN}

Inés María Mancini ${ }^{1}$

\section{Resumen}

El objetivo de este trabajo consiste en analizar los modos en los que los funcionarios del programa de prevención del delito Comunidades Vulnerables se insertan y comienzan a trabajar para prevenir la violencia en una villa de emergencia. Así, se trata de desentrañar cuál es la estrategia de trabajo en relación a los vínculos con la comunidad y a los supuestos que este trabajo implica acerca de la cohesión comunitaria y su relación con la prevención del delito.
PREVENTION OF CRIME IN A BUENOS AIRES SLUM (ARGENTINA). INTEGRATION AND PARTICIPATION, ANALYSIS OF COMMUNITY ASSUMPTIONS ON PREVENTION POLICIES

Inés María Mancini ${ }^{1}$

\begin{abstract}
:
The goal of this paper is to analyze the way in which people taking part in the Comunidades Vulnerables program work to prevent crime in a slum. In this way, this research reveals the action plan of this program in relation to the ties with community and the assumptions of this plan in relation to community cohesion and its relation with crime prevention.
\end{abstract}


Los resultados mostrarán que la perspectiva de la política pública parte del supuesto de que la transformación que los jóvenes deben experimentar para abandonar su posición de "conflicto con la ley" involucra la mejora de su posición en las relaciones comunitarias. Esto puede implicar la profundización de vínculos existentes o la generación de nuevos vínculos. Se procurará mostrar cómo estos supuestos entran en conflicto con ciertas prácticas de los actores, especialmente cuando la trama de relaciones cotidianas evidencia que los vínculos comunitarios también pueden estar atravesados por conflictos.

\section{PALABRAS CLAVE: PREVENCIÓN DEL DELITO, COMUNIDAD, JÓVENES.}

Fecha de recepción: 24.10.11

Fecha de aceptación: 23.03.12

1 Argentina. Licenciada en sociología (Universidad de Buenos Aires), magíster en antropología social (FLACSO Argentina). Doctoranda en antropología social Instituto de Altos Estudios Sociales, Universidad de San Martín (IDAES/ UNSAM). Becaria IDAES/UNSAM.

316 revista invi № 74 / Mayo 2012 / Volumen № 27: 315-341
The results of this research demonstrate that public policies are based on the assumption that young people should improve their position within community relationships to stop being in "conflict with law". This may imply the further development of existing links or the generation of new ties. This paper shows how these assumptions clash with certain practices of actors, especially when daily relationships demonstrate that community links can also be affected by conflicts.

\section{KEYWORDS: CRIME PREVENTION, COMMUNIT,} YOUNG PEOPLE.

Received: 24.10.11

Accepted: 23.03.12

1 Argentina. BA in Sociology (Universidad de Buenos Aires), MBA in Social Anthropology (ECLAC Argentina). Doctorate student in social anthropology, Instituto de Altos Estudios Sociales, Universidad de San Martín (IDAES/UNSAM). IDAES/UNSAM fellow. 


\section{Introducción}

En este trabajo, se analiza un aspecto específico del Programa de Comunidades Vulnerables: el lugar que la comunidad tiene en las estrategias de prevención social del delito.

Para comenzar, es preciso esbozar una breve descripción del programa ${ }^{2}$. El mismo se enmarca en el Plan Nacional de Prevención del Delito y fue diseñado a fines de 2001. Según la propia definición del programa, se trata de un modelo específico de abordaje territorial centrado en la integración social y la prevención del delito y la violencia. Sus objetivos consisten en garantizar el acceso de jóvenes de comunidades vulnerables ${ }^{3}$ a sus derechos fundamentales; incidir sobre los factores asociados a los procesos de vulneración social y reducir los índices de criminalidad, reincidencia y violencia ${ }^{4}$.

La ejecución del programa estuvo a cargo de la Dirección de Política Criminal del Ministerio de Justicia y Derechos Humanos de la Nación (Argentina). En algunos territorios, cada gobierno local ejecutaba el programa siendo asistido y supervisado

2 Para una descripción del funcionamiento del programa desde la perspectiva de sus funcionarios, véase Ciafardini, Mariano, 2005.

3 A lo largo de este escrito se indicarán en itálicas los términos nativos, tanto cuando correspondan a la terminología utilizada en el contexto del programa y de sus funcionarios como aquellos términos nativos empleados por los habitantes de la villa de emergencia. Asimismo, se utilizarán las comillas para las citas textuales. por funcionarios del gobierno nacional, mientras que otros eran trabajados mediante la inserción de operadores del Estado nacional. En 2008, el programa dejó de desarrollarse en el ámbito nacional y, desde entonces, sólo continuaron trabajando los gobiernos locales que lo consideraron pertinente.

Además, el programa que estudiamos funcionaba en una oficina propia y alejada de otras oficinas correspondientes al Plan Nacional de Prevención del Delito. Desde la perspectiva de los funcionarios del programa, esto no era más que una demostración física de su lugar periférico dentro del Estado. Pero este posicionamiento los distinguía, desde su perspectiva, de otras políticas estatales y su lugar periférico era referenciado con orgullo, en tanto y en cuanto entendían que era producto del corte progresista de la política que ejecutaban. Al mismo tiempo, ello implicaba que estos funcionarios experimentaran ciertas dificultades para gestionar recursos necesarios para los beneficiarios frente a otras dependencias del Estado. En general, estas articulaciones se lograban gracias a contactos personales. 
En la práctica, el funcionamiento del programa implicaba la asistencia de operadores a una comunidad vulnerable, o territorio en donde se realizaban diversas actividades con los beneficiarios del programa. Las mismas eran variadas: había talleres sobre distintas temáticas propuestas por el programa (como por ejemplo, un taller de identidad), también se abordaban problemáticas que los operadores consideraban pertinentes para un grupo de beneficiarios en particular (como por ejemplo, talleres sobre búsqueda de empleo, organización del tiempo, o género) y se producían salidas o actividades puntuales por demandas específicas de los beneficiarios.

Desde los documentos que presentan el programa, se enunciaba un conjunto de rasgos específicos para seleccionar los posibles beneficiarios, de modo de distinguir la estrategia social preventiva de otros planes sociales. Así, se postulaba que se debía apuntar a jóvenes entre 15 y 25 años que hubieran estado involucrados en prácticas violentas próximas al delito o que tuvieran una causa judicial abierta relacionada con actos de violencia y delitos callejeros o que hubieran estado en instituciones de encierro o que registraran al menos un ingreso a la comisaría en el último año ${ }^{5}$. Además, se define a estos beneficiarios como beneficiarios directos en tanto y cuanto son quienes participan

5 Ciafardini, Mariano, 2005.

318 revista invi № 74 / Mayo 2012 / Volumen № 27: 315-341 activamente de las actividades del programa y perciben un beneficio económico. Y se entiende que los familiares, vecinos y amigos de los beneficiarios se constituyen como beneficiarios indirectos, dado que adquieren nuevas perspectivas que los beneficiarios directos estarán en condiciones de aportar.

Un aspecto central a tener en cuenta en este análisis es que el programa articula sus acciones con el Ministerio de Trabajo, permitiendo que los beneficiarios perciban un Plan de Empleo Comunitario ("el PEC") cuyo monto alcanzaba los $\$ 150^{6}$ mensuales y se cobraba en una entidad bancaria. Resulta fundamental considerar esta cuestión puesto que el dinero funciona como regulador de la relación entre operadores y beneficiarios. Así, uno de los efectos más notorios (tanto desde la perspectiva de los beneficiarios como de los operadores) de la desvinculación del programa consiste en dejar de percibir este ingreso mensual.

Los datos empíricos retomados en este trabajo se referirán a la observación de un grupo de operadores asignados a una villa de emergencia situada en la ciudad de Buenos Aires.

En esta villa, el programa era desarrollado tanto por operadores del gobierno nacional como por operadores del gobierno de la Ciudad de Buenos

6 En 2005, a comienzos de mi trabajo de campo, esta suma equivalía -aproximadamente- a 52 dólares y descendió a 47 dólares hacia fines de mi trabajo de campo en 2007. Inserción y participación, análisis de los supuestos de comunidad en las políticas de prevención / 
Aires. Los mismos operadores vinculados al gobierno nacional que coordinaban el trabajo con un grupo de beneficiarios supervisaban el trabajo de los operadores del gobierno local que trabajaban con otro grupo de jóvenes. Cuando el programa dejó de desarrollarse en el ámbito nacional, los beneficiarios comenzaron a trabajar con operadores del gobierno de la ciudad.

El trabajo de campo fue realizado entre 2005 y 2007 (con algunas revisitas posteriores) en una villa de emergencia de la Ciudad de Buenos Aires. Allí, los operadores nacionales del programa organizaban reuniones con un grupo de alrededor de veinte beneficiarios dos veces a la semana.

En una primera instancia se realizaron entrevistas con funcionarios del programa, quienes me introdujeron al equipo de trabajo de operadores asignados a esta villa. Luego de un tiempo, los operadores me permitieron acceder a la mayoría de las actividades que realizaban: reuniones en la oficina y en bares, me participaron del intercambio de correos electrónicos y me permitieron acceder a distintas documentaciones relacionadas con el programa. Además, pude acceder a las reuniones que se realizaban con los beneficiarios. Por otra parte, mi presencia sostenida en la villa me permitió entrevistar a beneficiarios y otros residentes.

Cuando comencé mi trabajo de campo las reuniones tenían lugar en un salón facilitado por una Iglesia católica de la villa. El mismo estaba ubicado en la parte trasera de la iglesia y era utilizado para celebraciones de casamientos o bautismos, velorios o reuniones de distinta índole ${ }^{7}$. Sin embargo, esta sede era transitoria, puesto que las reuniones solían organizarse en un comedor, que se encontraba en obras para la ampliación de sus instalaciones. Cuando se produjo un intento de robo a algunos operadores en las inmediaciones de la iglesia, consideraron oportuno retornar al comedor (ubicado en el extremo opuesto de la villa), pese a que las refacciones aún no estaban finalizadas.

A las reuniones del programa, las mujeres iban acompañadas por sus hijos o por hijos de vecinas que trabajaban, que eran cuidados a cambio de una remuneración diaria. Así, los encuentros se caracterizan por un bullicio permanente: conversaciones superpuestas, llantos y gritos de niños.

7 Esto implicaba que, en algunas ocasiones, el espacio no estaba disponible en el horario estipulado, pues era requerido para alguno de sus otros usos. Además, como era utilizado para velorios en algunas ocasiones, las jóvenes se negaban a tener la reunión allí puesto que sostenían que había "olor a muerto" y que esto podía ocasionarles pata de cabra a los niños. 
Cuando comencé mi trabajo de campo el grupo de operadores estaba compuesto por tres funcionarios: Osvaldo, Lucas y Ernesto. Además, participaban, aunque ocasionalmente, dos voluntarias. Después del primer receso ${ }^{8}$ que presencié, el grupo de operadores cambió. Osvaldo volvió a su trabajo en rehabilitación de adicciones y se incorporó María Clara. Algunos meses más tarde, comenzó a trabajar Luciana en el equipo.

Por su parte, el grupo de beneficiarios fue modificándose bastante a lo largo del tiempo en el que realicé mi trabajo de campo. En general, el grupo contó con alrededor de veinte beneficiarios y con una cantidad similar de varones y mujeres. Si bien el diseño del programa establecía que la edad debía estar comprendida entre los 15 y 25 años, en la práctica, los beneficiarios tenían al menos 16 años, puesto que esa era la edad mínima para poder cobrar el PEC y eran pocos los casos de jóvenes en el extremo superior de la franja establecida.

Además, eran pocos los casos de jóvenes mayores de 22 años que formaban parte del programa, lo

8 Durante el mes de enero el programa no realizó actividades en la villa.

320 revista invi № 74 / Mayo 2012 / Volumen № 27: 315-341 cual obedecía al "conocimiento informal" que media entre la prescripción de las instituciones y la práctica ${ }^{9}$, pues se suponía que no era posible trabajar con los mayores por la posibilidad de que sus carreras delictivas estuvieran más consolidadas.

Como el reclutamiento se hacía, en buena medida por recomendaciones de los propios beneficiarios o de las encargadas del comedor, era frecuente que dentro del programa hubiera parientes (hermanos, primos o sobrinos) o grupos de amigos. Esto implicaba que algunas disputas familiares pudieran tener lugar también dentro de las reuniones del programa ${ }^{10}$ o que el programa se hiciera presente en el seno de la vida familiar ${ }^{11}$.

Así, el trabajo del programa se desarrolla en un lugar particular: una villa de emergencia. Sobre este tipo de lugares existen afirmaciones de sentido común, periodísticas, artísticas y científicas que trabajan sobre la relación entre villa y delito. Por ello, resulta fundamental esbozar una descripción del espacio que nos permita entender cómo se inserta el trabajo de un programa de prevención del delito.

$9 \quad$ Esto es señalado por Míguez, Daniel, 2008.

10 Por ejemplo, en una ocasión se registraron disputas en las reuniones cuando una beneficiaria, prima de otra, le "robó el marido".

11 En una oportunidad, un beneficiario me contó que había tenido una pelea con su tía, quien lo había recomendado al programa, y que en medio de la pelea ella le habría dicho "le voy a decir a Ernesto que te saque del plan". 
La villa ${ }^{12}$ está ubicada en el distrito más próspero del país: la Ciudad de Buenos Aires. Se trata de una de las villas más pobladas de la ciudad. Las estimaciones realizadas a partir del censo del año 2010 sugieren que tiene una población de 25.973 personas (de las 163.587 que viven en villas en la ciudad) $^{13}$. Al mismo tiempo, muchos medios de comunicación la han catalogado como una de las más "peligrosas", recurriendo a comparaciones con las favelas brasileras o señalando un proceso de favelización, lo cual implica hablar de una supuesta ausencia del Estado.

Como suele ocurrir con este tipo de enclaves, su posicionamiento en la ciudad es periférico. El viaje desde cualquier barrio de la ciudad considerado de clase media, muestra una desconexión que resulta bastante peculiar: así como resulta posible para casi cualquier habitante de la ciudad de clase media realizar sus recorridos cotidianos sin atravesar la villa, cuando se llega a ella sorprende su cercanía con otros barrios de clase media y media alta y su conexión (tal vez algo dificultosa pero existente) a partir de medios de transporte.

La villa está delimitada por grandes avenidas de circulación rápida y varios espacios verdes privados

12 Nos referiremos al lugar sin mencionar su nombre como "la viIla", tal como la llaman muchos de sus habitantes para proteger la confidencialidad de los consultantes. Por el mismo motivo, todos los nombres de los informantes citados son ficticios. (clubes, campos de deportes, etc.) Esta condición refuerza la idea de aislamiento. Por las avenidas, circulan varios colectivos ${ }^{14}$ (aproximadamente trece líneas) ${ }^{15}$ que permiten acceder a distintos puntos de la Ciudad. Sin embargo, las distancias entre muchas de las casas y las avenidas es muy grande y ningún medio de transporte atraviesa la villa, pese a que hay algunas calles abiertas que permitirían su circulación.

Por lo general, las casas tienen ladrillo a la vista y muchas de ellas tienen más de una planta. La mayoría cuenta con rejas en todas sus aberturas e incluso se observan rejas en algunos pasillos. Esto implica que los vecinos que habitan las casas de un pasillo tienen las llaves, mientras que el resto no puede circular por ese espacio.

También, hay algunos edificios de vivienda social que, en teoría, constituyen barrios ${ }^{16}$ con nombre propio, pero en la práctica se perciben como parte de la villa, salvo en situaciones de conflictos específicos.

Más allá de las percepciones del espacio de sus habitantes, fundadas en un conocimiento de las relaciones sociales, una descripción de las condiciones físicas debe notar la diferencia entre estos edificios de vivienda social que muestran construcciones

14 Además, algunos de esos colectivos permiten acceder a una línea de subterráneo en menos de quince minutos.

15 Gutierrez, Andrea y Rearte, Julio, 2006.

16 En el número de población presentado más arriba no se considera la población que habita en estos barrios. 
regulares, calles asfaltadas, instituciones (como una de las salitas) de aspecto más moderno, y aquellas zonas de pasillos intrincados y angostos entre los que resulta difícil ubicarse. En muchas de estas zonas de pasillo, las calles son de tierra y algunas resultan inundadas en casos de lluvias muy fuertes. La precariedad de ciertas construcciones, sumadas a las condiciones geográficas (se trata de un terreno bajo), implica que las vidas de los residentes estén sujetas a frecuentes problemas de humedad, inundaciones, problemas con las cloacas, la recolección de basura e incendios. En ocasiones estas cuestiones son fuente de conflictos entre vecinos, pero tal como sostiene Juhem ${ }^{17}$, no podemos atribuir todos los fenómenos de violencia al ambiente urbano degradado, sino que el aglutinamiento de personas en un espacio reducido, implica una gran concentración de varones adolescentes y con ello el desarrollo de una cultura de la calle ${ }^{18}$.

Caminar por las calles o pasillos de la villa durante la mañana o las primeras horas de la tarde, da una sensación completamente diferente a la que se tiene cuando esto se realiza en las últimas horas de la tarde o por la noche. Mientras que una caminata temprana nos da la impresión de ser un lugar con una elevada presencia femenina, la noche da

17 Juhem, Philippe, 2000.

18 Más allá de si estas afirmaciones son generalizables o si pueden sostenerse para el barrio abordado, son consistentes con la perspectiva con la que trabaja el programa. la impresión opuesta: grupos de varones tomando cerveza o jugando a la pelota en todas partes.

En general, los almuerzos se hacen en comedores de distinta índole (hay comedores de las diferentes iglesias, comedores creados por vecinos con o sin apoyo estatal). Algunos tienen mesas y quienes están anotados comen allí, mientras que otros entregan la comida. En cualquier caso, una caminata al mediodía muestra gente haciendo filas en diferentes puntos del barrio.

Dentro de la villa, hay comercios que pueden abastecer las necesidades inmediatas de todos los vecinos: kioscos, almacenes, bares y -el negocio predominante- locutorios con Internet.

También hay escuelas, de distintos niveles, que permiten que la mayoría de los estudiantes cursen su educación dentro del barrio. Además, hay dos salas de atención médica que funcionan durante el día.

Todos estos servicios permiten que buena parte de sus habitantes resuelvan dentro de la villa sus necesidades inmediatas. Si además tenemos en cuenta que hay una gran cantidad de habitantes que carecen de empleos formales, podemos concluir que la cotidianidad trascurre para la mayoría de los vecinos dentro del barrio. Ello da lugar a una 
intensificación de las relaciones cara a cara entre los residentes.

Por último, queremos destacar un dato sobre la población de la villa. Nos referimos a la convivencia de argentinos, bolivianos, peruanos y, en menor medida, paraguayos. Es posible notar una cierta tendencia al agrupamiento de viviendas según las nacionalidades. En ocasiones, algunos de los conflictos que tienen lugar entre vecinos pueden explicarse en virtud del particular procesamiento de estas diferencias.

\section{¿Por qué trabajar con jóvenes villeros? Privaciones y transformación}

Como mencionamos al comienzo, nuestro estudio está enfocado en el desarrollo de una política de prevención del delito; se trata de la voluntad del Estado de anticiparse a la producción de ciertos hechos. Sozzo ${ }^{19}$ analiza las estrategias políticas implementadas desde los gobiernos locales para reducir el delito en la Argentina. De modo esquemático, el autor reconoce tres tipos de intervenciones: la estrategia situacional ambiental, la estrategia social y la estrategia comunitaria. El programa estudiado se enmarca en lo que denominamos estrategia social. Para comenzar, hay que señalar

19 Sozzo, Máximo, 2009 que "se trata de un conjunto complejo de intervenciones que buscan afectar los procesos sociales y culturales que se conciben como forjadores de condiciones de posibilidad para que un individuo se comprometa en la realización de actividades delictivas"20

Dicha estrategia se ha ido construyendo a partir del saber criminológico. Sin embargo, sus presupuestos no pueden ser pensados de modo unívoco, por el contrario, Sozzo identifica dos vertientes teóricas. Una de ellas entiende que la criminalidad de la calle tiene que ver con un déficit cultural de quienes producen los delitos. Desde esta perspectiva, resulta fundamental producir modificaciones en ciertos espacios de formación del control social y del autocontrol: la familia, la escuela, el grupo de pares. La otra vertiente entiende al déficit relacionado con privaciones materiales.

La propuesta del programa parece poder enmarcarse en las dos vertientes de la prevención social. En primer lugar, se trata de que ese espacio antes señalado de la cultura de la calle se modifique hasta dejar de ser forjador de condiciones para la producción de delitos, al mismo tiempo que se trabaja para que los jóvenes establezcan otros vínculos: vínculos comunitarios, con la escuela, con ciertos familiares. Respecto de la segunda vertiente, la entrega de un dinero mensual pretende sortear

20 Ídem, p. 66 
las dificultades materiales inmediatas, pese a que se trata de una suma de dinero que no puede ser comparada con los beneficios económicos que se obtienen a partir de ciertas actividades ilegales.

Por otro lado, es conveniente aclarar, como lo hace Sozzo, que estos presupuestos teóricos no son necesariamente conocidos o compartidos por quienes aplican los programas, ya que los mismos han sido traducidos a un lenguaje político y circulan más allá de las justificaciones teóricas que los originan.

De todas maneras, parece compartido por quienes ejecutan o proponen este tipo de políticas el supuesto de que la producción de este tipo de delitos (menores) se relaciona con algún tipo de privación. Así, la prevención debería centrarse en la inclusión de los actores, brindándoles acceso a ciertos capitales que les están vedados en la estructura social.

Por otro lado, creemos pertinente señalar que además de esta disociación entre teoría y política, existe una distancia entre los postulados de quien diseña la política, quienes la dirigen y quienes la aplican efectivamente en el territorio. Sin embargo, la idea de exclusión parecería poder ser pensada como un punto en común entre todos ellos. Por lo tanto, son consideradas pertinentes las acciones que tienden a la inclusión.
Ahora bien, la inclusión de los jóvenes que daría respuesta a esta privación, sólo puede producirse si los beneficiarios del programa modifican ciertos aspectos de sus vidas. De este modo, se les propone, con mayor o menor grado de explicitación, que inicien un proceso de transformación.

Y uno de los aspectos que se considera que los jóvenes deben invertir, uno de los sentidos en los que deben transformarse involucra la profundización de sus vínculos positivos y la merma o abandono de malas compañías (que desde la perspectiva de los beneficiarios y vecinos del barrio son las juntas).

Las reflexiones en torno al concepto de transformación o conversión no serán profundizadas en este trabajo. Sin embargo, parece pertinente detenerse en el hecho de que es una idea inherente a muchas políticas sociales y que el hecho de que el cambio involucre a los vínculos de los sujetos de intervención parece reiterarse. Sólo a modo de ejemplo, encontramos en el trabajo de Bufarini ${ }^{21}$ sobre un programa municipal de asistencia en Rosario a personas que viven en la calle la estrategia de la restauración de vínculos afectivos con familiares o amigos para evitar la cronicidad. 


\section{De recursos e intercambios}

Hasta ahora hemos enunciado una serie de características básicas que pretenden esbozar una idea del programa y de su inscripción en el aparato administrativo y en ideas acerca de la prevención del delito. Consideramos necesario hacer hincapié en los recursos con los que el programa cuenta para funcionar para luego poder problematizar algunas de las cuestiones que anticipan los análisis de Sozzo así como también profundizar en su funcionamiento.

En principio, el programa requiere para su funcionamiento de dos actores básicos: los operadores y los beneficiarios directos. Como veremos, estos no son los únicos actores, pero sin ellos el programa resulta impensable. Además, esta interrelación entre operadores y beneficiarios directos se sostiene -en mayor o en menor medida- por el recurso del dinero.

Ahora bien, el vínculo inicial con los beneficiarios directos no se genera de modo espontáneo, sino que debe haber un modo en el que estos actores básicos se conozcan y decidan iniciar un trabajo conjunto. En este sentido, el sistema judicial funciona como un actor del programa en la medida en que los jóvenes judicializados o recién salidos de prisión suelen ser derivados a participar en este programa.
Sin embargo, la modalidad de reclutamiento más frecuente es la recomendación de beneficiarios o de otros vecinos del barrio que colaboran con el programa. Estos vecinos a los que los operadores consultan son definidos por el programa como actores comunitarios. En el caso de la villa que estudiamos se trata principalmente de las encargadas de un comedor y, en menor medida, del cura de una iglesia que facilitan los lugares en los que se realizan las reuniones del programa e introducen potenciales beneficiarios a la consideración de los operadores, al mismo tiempo que les proporcionan distintos tipos de información a los operadores sobre los beneficiarios y sobre el barrio en general.

Estos actores comunitarios o referentes son personas en las que los operadores por lo general creen y que consideran habilitadas para informar acerca de los vínculos de los beneficiarios con sus familiares o amigos. Además, los operadores organizaron una asamblea multifamiliar (la multi) a la que asistían parientes o vecinos de los beneficiarios directos para hablar de los problemas de los chicos del barrio.

Con todos estos recursos, se produce un intercambio y una circulación de dinero, información, mecanismos de resolución de problemas, prestigio. Si bien esta circulación no se verifica siempre en la modalidad originalmente planificada por los operadores, queremos destacar que ella no se da en cualquier dirección. En efecto, la intención de 
conocer información acerca de los vínculos de los beneficiarios o la selección de actores comunitarios implica el supuesto de que hay vínculos positivos y negativos, gente que se preocupa por el barrio y participa y gente que es indiferente.

Entre los recursos con los que cuenta el programa, el dinero es el más saliente del intercambio, sin embargo no es menos cierto que sin otros recursos el programa no se sostiene, lo cual implica que no continuaría el trabajo de los operadores. De este modo, todos los actores tienen algún tipo de interés en este intercambio. En efecto, si no hay beneficiarios, los operadores no pueden realizar su trabajo. Cabe destacar que todos los actores pueden obtener -además- el beneficio de sentir que están obrando bien y haciendo lo mejor por el barrio por el solo hecho de participar de esta relación.

Como se mencionó anteriormente, para el reclutamiento de beneficiarios, los operadores dependen -en buena medida- de sus relaciones con los actores comunitarios que harán de nexo para presentar a potenciales beneficiarios. A su vez, los actores comunitarios son beneficiados porque su relación con los operadores les permite acceder a información y gestiones. Nos referimos a lo que la literatura especializada en clientelismo político ${ }^{22}$ ha catalogado de cadenas de resolución de problemas, ubicando

22 Auyero, Javier, 1997

326 revista invi № 74 / Mayo 2012 / Volumen № 27: 315-341 a ciertas personas en el lugar de mediadores. Más allá de que esta perspectiva de clientes y patrones no se corresponda con el punto de vista nativo ${ }^{23}$, mencionamos que el mecanismo en el que estas relaciones se sustentan se corresponde con lo que la literatura teórica ya ha clasificado de ese modo.

Ahora bien, es preciso enfatizar que ninguno de los actores involucrados en estas redes de intercambio acordaría en que estas cadenas de resolución de favores pueden ser denominadas como clientelismo político. Por el contrario, puntero es un calificativo que le cabe a otras personas que no están inscriptas en la red del programa: para los operadores los actores comunitarios son referentes, mientras que otras personas que trabajan en la villa pueden ser pensadas como punteros.

Para comprender mejor qué es lo que se intercambia o cuáles son los recursos que ponen en juego, puede ser útil describir cómo se generó la relación entre los operadores y sus actores comunitarios, las chicas del comedor ${ }^{24}$, entre quienes Mariana se destaca pues es quien organiza el trabajo de sus compañeras y también quien tiene una relación más estrecha con los operadores. Este relato puede servir para comprender cómo la relación entre operadores y actores comunitarios está marcada por ciclos de confianza y reciprocidad.

23 Frederic, Sabina, 2004

24 De este modo se refieren los operadores a las encargadas del comedor. 
El contacto inicial con Mariana fue realizado por las coordinadoras del programa. Por lo que me relataron tanto Mariana como las coordinadoras, la reacción inicial fue de desconfianza: a Mariana le parecía extraño que el Ministerio de Justicia quisiera hablar con ella y a las coordinadoras se les dificultaba explicar lo que querían hacer, hasta que confió en ellos y su hija fue una de las primeras beneficiarias. El vínculo de Mariana con el programa creció hasta el punto de que en la reunión de despedida les dijo llorando a los operadores y a los coordinadores: "ustedes salvaron a mi hija".

La idea de que el inicio de la relación del operador con el barrio en general y con actores comunitarios en particular parte de la desconfianza está explicitada en la documentación del programa ${ }^{25}$ y es un punto de partida en la labor práctica. Desde la perspectiva de operadores y otros funcionarios del programa, es a través de la constante presencia en el barrio que se construye la confianza.

No hay un programa específico para generar relaciones de confianza, sino que los operadores gestionan vínculos con actores comunitarios y con vecinos del barrio que acuden espontáneamente a pedir ayuda de distintas clases mientras los operadores están llevando a cabo reuniones con los beneficiarios. Estos vecinos pueden ser parientes de los

25 Así, Ciafardini sostiene que la gente está cansada de los experimentos de toco y me voy y que por este motivo le tomará examen al operador muchas veces. beneficiarios que piden asesoramiento para resolver temas de los jóvenes o padres de otros jóvenes del barrio, pero también viene gente a pedir que nada tiene que ver con los objetivos declarados del programa: asesoramiento respecto a problemas laborales como despidos, cuestiones administrativas como cobros de pensiones o programas sociales específicos, conflictos con vecinos, problemas relacionados con la documentación de hijos o nietos, etc.

Si bien el cargo de operador no exige una formación profesional en particular, el hecho de que algunos de ellos fueran abogados, les proporciona un saber específico que los coloca en la posición de poder dar alguna orientación a la mayoría de estas demandas espontáneas. Sin embargo, este saber específico no está en exclusiva relación con el título de abogado, sino que hay un saber más general respecto del manejo de distintas instancias burocráticas que les posibilita resolver algunos problemas de los vecinos del barrio.

Ahora bien, las posiciones de cada uno de los actores en esta red de intercambios no son equivalentes: no todos obtienen el mismo caudal de beneficios. Los beneficiarios obtienen un ingreso mensual, los operadores obtienen una de las justificaciones de su propio empleo (lo cual implica también un ingreso mensual), pero los beneficios 
de los actores comunitarios no se producen de modo permanente y se verifican ciertas situaciones en las que estos actores son sometidos a demandas, sin obtener -aparentemente- nada a cambio. Pese a ello, se sigue observando una retórica de reciprocidad. Sucede que, tal como señala Herzfeld ${ }^{26}$, las desigualdades no pueden abolir la retórica de la reciprocidad. Así, en muchas partes del mundo, las inequidades del patronazgo están marcadas por la parafernalia de la reciprocidad.

Este tipo de relaciones son provechosas de ser estudiadas cuando quiere analizarse el rol de la comunidad puesto que nos llevan a la pregunta por el lazo social. En efecto, se trata de relaciones que no son puramente contractuales, lo cual en términos de la teoría clásica ${ }^{27}$ es lo que haría entrar en crisis al lazo social. Sin embargo, cabe preguntarse si efectivamente la existencia de este tipo de lazo social basado en la construcción de relación de confianza (aparentemente escindidas del economicismo) genera un lazo social duradero, al que además se le pedirá solución al problema del delito.

Así, tal como lo señala Fonseca ${ }^{28}$, los líderes comunitarios que representan al barrio ante los poderes municipales y estatales son objeto constante de chismes. Por ejemplo, se dice que los alimentos que se dan a los centros comunitarios para

26 Herzfeld, Michael, 1993.

27 Mauss, Marcel, 1966.

28 Fonseca, Claudia, 2004 distribuirse gratuitamente aparecen a la venta, etc. Hallazgos similares fueron detectados en nuestro trabajo de campo ${ }^{29}$. Entonces, cabe preguntarse qué ocurre con la construcción de este vínculo y con la confianza y, especialmente, la pregunta que nos compete es si el Estado al contribuir a minar la confianza con la que trabajan los actores comunitarios no está al mismo tiempo socavando sus propios propósitos de prevenir el delito al trabajar sobre ciertos vínculos considerados positivos.

\section{Los operadores: emprendedores morales y su inserción en la comunidad}

Nos interesa detenernos brevemente en la posición de los operadores y en el análisis de las consideraciones teóricas que pueden hacerse de esta inserción en el campo. Desde la perspectiva de Becker ${ }^{30}$, los operadores podrían ser considerados en ciertos momentos como emprendedores morales, en tanto y en cuanto son quienes aplican y en ocasiones crean normas. Siguiendo, los postulados de este autor señalamos que "(e)s muy interesante el hecho de que la mayor parte de la investigación científica y la especulación sobre la desviación se ocupen más de las personas que rompen las normas que

29 Mancini, Inés, 2009

30 Becker, Howard, 2009. 
de quienes las producen o aplican. Para lograr una comprensión cabal del comportamiento desviado debemos mantener el equilibrio entre estos dos posibles enfoques de investigación"31.

Dentro de las oficinas del programa, las relaciones eran estrechas e intensas y se observaban por lo general armoniosas relaciones entre colegas. De todas maneras, era posible identificar algunas heterogeneidades que podían ser fuente de conflicto. En ocasiones, algunos de sus operadores eran acusados de sobreimplicarse en los casos de ciertos jóvenes por el resto de los funcionarios del plan. Esta crítica aludía al "exceso" de acompañamiento a ciertos jóvenes que les pedían a los operadores verlos durante el fin de semana o que intervinieran en sus asuntos de modo tal que el resto del equipo entendía que se tomaba el lugar de la familia del joven. Para los "acusados", esta crítica estaba sesgada por el hecho de que muchos de los "acusadores" no trabajaban en territorio, por lo cual no comprendían que, en ocasiones, el trabajo implicaba involucrarse cuerpo a cuerpo.

Al mismo tiempo, dentro del grupo de operadores asignado a la villa, también se registraron conflictos por diferencias de criterio y de niveles de entusiasmo frente al trabajo con los jóvenes.

Nos interesa señalar que la posición de los operadores es por demás compleja. En principio, resultaría sencillo adjudicarles la intención de disciplinar a los beneficiarios, o imputarles las fallas del programa en imponer o hacer cumplir ciertas reglas y, sobre todo, en transformar a los jóvenes en personas socialmente integradas. Sin embargo, queremos destacar que los problemas o contradicciones sobre los que trabajaremos tienen lugar más allá de las buenas intenciones y el compromiso que los operadores sostienen y de las relaciones de afecto que entablan con los beneficiarios y vecinos de la villa.

Sólo para esbozar las dificultades a las que está sujeta su posición enunciaremos algunos problemas en las interacciones con otros actores y entre ellos. Como se mencionó anteriormente se verifica una fractura básica relacionada con la acusación de sobreimplicación que realizan otros miembros del programa. Sin embargo, estas acusaciones soslayan el hecho de que al mismo tiempo que se recibe esta imputación, los operadores son requeridos por los vecinos del barrio a implicarse, a comprometerse. Y también se deja de lado el hecho de que el mismo programa para funcionar requiere que los operadores construyan vínculos de confianza, de los cuales es lícito suponer que podrían surgir demandas de compromiso y presencia.

Uno de los casos más conflictivos que presencié en este sentido se relacionó con el modo en que los operadores reaccionaron frente a un beneficiario,

31 Ídem, p. 184 
Diego, que consumía drogas, cometía ocasionalmente delitos menores dentro del barrio, tales como robar en los fines de semana en una feria en la que se vendían diversos productos. La situación de Diego se mostró crítica para los operadores el día que intentó suicidarse. A partir de entonces, los operadores realizaron gestiones para que se internara en distintas instituciones, ya que se escapó de más de una. Además de estas gestiones, algunos operadores fueron a visitarlo mientras estaba internado, hablaron con sus familiares, y lo buscaron intensamente en una de sus huidas. A partir de estas intervenciones fueron acusados de sobreimplicarse, de no darle lugar a la familia del joven y hasta se les prohibió seguir interviniendo. Esto implicaba que no podían visitarlo ni realizar gestiones institucionales, ya que estas cuestiones eran consideradas tareas que debía realizar la familia. Una vez que esto ocurrió y que los operadores me comentaron esta situación, se acercó la madre de Diego a una operadora para consultar algunas cuestiones. María Clara le dio el teléfono de la institución y le dijo que ella debía llamar. La madre de Diego tomó en sus manos el papel que le daba María Clara y le dijo "es que yo no sé hablar". Frente a esta respuesta, María Clara tomó su celular y llamó ella misma a la institución, resolviendo el problema. Cuando subimos a su auto me dijo que las críticas que habían recibido eran infundadas y que ella como toda peronista ${ }^{32}$ se consideraba verticalista y obedecía reglas, pero que -llegado el caso- cuando debía violar alguna regla, violaba todas juntas.

Este caso resultó tan particularmente conflictivo que suscitó la realización de un ateneo en el que todos los miembros del programa debatieron lo sucedido. Como no pude asistir, luego de leer los intercambios registrados en la reunión, me comuniqué con Ernesto para preguntarle cómo lo había vivido y lo primero que me señaló fue que había sido un buen encuentro porque finalmente se había entendido la participación que habían tenido.

La breve descripción de este caso nos resulta útil para señalar que la posición del operador es compleja y está sujeta a demandas diversas y contradictorias. El trabajo de Marlow y Pitts ${ }^{33}$ señala que las contradicciones que viven los trabajadores en barrios comparables de Inglaterra implican que muchos profesionales se sienten aislados, devaluados y proletarizados. Sus investigaciones encuentran que los profesionales no se sienten valorados por los políticos y que creen que su servicio no puede remediar los problemas que enfrentan. Muchas veces, estos malestares se traducen en ausentismo del staff y renuncias.

32 Aludiendo a su identificación con el partido justicialista en la Argentina.

33 Marlow, Alan y Pitts, John, 1999. 
Se trata -desde cualquier punto de vista- de un trabajo que presenta exigencias emocionales cuando se produce el compromiso mínimo que se requiere para establecer esas relaciones de confianza con los residentes. Sin embargo, no todos los operadores que trabajan en este barrio toman el mismo nivel de compromiso. Tanto Ernesto como María Clara son considerados por todos como más comprometidos y conciben su trabajo en términos de militancia. En muchas ocasiones los propios colegas describen a María Clara como alguien a quien su trabajo le cuesta "hasta la salud".

Consistentemente, los beneficiarios y los actores comunitarios muestran una mayor tendencia a plantear sus problemas a los operadores más comprometidos, con lo cual el nivel de demanda que estos reciben se ve reforzado.

Estos diversos niveles de compromiso y dedicación y otras divergencias en la metodología de trabajo producen también fracturas internas en el grupo de operadores que trabajan en la villa. En ocasiones, estas diferencias se hablan abiertamente mientras que en otras, las acusaciones recíprocas son veladas.

Esta exigencia emocional intrínseca al tipo de trabajo desarrollado se hace manifiesta en distintas oportunidades. Las reiteradas imposibilidades de cumplir con las consignas de trabajo impuestas por ellos mismos podrían leerse en este sentido: llegadas tarde, faltas y, en algunos momentos, la declarada imposibilidad de ir a terreno como método de autopreservación, que es avalado por el resto de los colegas. De este modo, podríamos señalar que se trata de un trabajo de una gran exigencia pero que al mismo tiempo posibilita ciertas libertades y deja espacio para tomar decisiones como las de asistir o no, de modo relativamente autónomo.

Estas exigencias a las que está sometido el trabajo de los operadores se relacionan en buena medida con la producción de demandas contradictorias. La propia inserción en el barrio y la generación de la relación de confianza con los actores comunitarios que da pie al inicio del programa los puede llevar a ser cuestionados "desde arriba" por otros funcionarios políticos. Así, se trata de un enigma sin resolver: si se descuidan las relaciones con los actores comunitarios y se quiebra esa confianza, se socavan los pilares del trabajo en la comunidad, si no se atiende a las observaciones hechas por otros funcionarios políticos, se corren riesgos respecto del propio trabajo. En este sentido, podemos comprender la sensación de aislamiento a la que hacíamos mención más arriba. 


\section{Los actores comunitarios: comunidad y prevención del delito}

Como ya se mencionó, la inserción del programa en el barrio se da a partir del trabajo de operadores que se contactan en primer lugar con los actores comunitarios que son los que trazan las relaciones con los potenciales beneficiarios y los que facilitan instalaciones dentro del barrio para llevar a cabo las reuniones. Además, se espera que estos actores faciliten información sobre los beneficiarios, sus vínculos y los acontecimientos generales del barrio, especialmente aquellos vinculados al delito.

Cabe destacar que las expectativas depositadas en estos actores se sustentan en el mero hecho de compartir la preocupación por nuestros chicos, por el barrio. Se parte entonces del supuesto de que la comunidad rechaza la violencia y desea la transformación de los jóvenes en potencial peligro.

A continuación, haremos algunas consideraciones relacionadas con la literatura del desarrollo que resultan pertinentes para comprender y reflexionar acerca del rol de los actores comunitarios en el programa. Nos referiremos a ideas muy trabajadas por esta literatura y que, al mismo tiempo, tienen algún tipo de resonancia en la práctica del programa o en los relatos de los operadores. Así, es frecuente que los operadores se refieran al empoderamiento de ciertas personas o que se demande la participación de los vecinos.

Ahora bien, en este tipo de conceptos subyace una idea que se relaciona con la concepción -se lo explicite o no- de comunidades cohesionadas sin la existencia de divergencias y conflictos. Y esta idea aparece en ocasiones en los discursos y prácticas de la intervención hasta que los conflictos emergen de modo estridente o simplemente cuando los jóvenes manifiestan que no van a emprender determinadas tareas comunitarias solicitadas por los operadores porque beneficiarían a "los bolitas"34.

Pese a lo dicho, es justo reconocer que el propio programa pregona reflexiones frente a esta lectura inocente de la comunidad. Así, Ciafardini ${ }^{35}$ critica la existencia de un concepto mítico de comunidad en el que se supone la existencia de un consenso moral. En este sentido, reconoce que los territorios urbanos son agregados sociales complejos en los que existe una conflictividad intracomunitaria, con voces diversas y señala la importancia de la participación de las distintas voces.

Si bien es cierto que en la práctica no se trabaja con un supuesto de comunidad homogénea, no a todas las voces se les atribuyen los mismos roles en este

34 Modo despectivo de referirse a los bolivianos.

35 Ciafardini, Mariano, 2005. 
juego comunitario. Y esto no parece ser un sesgo de la práctica frente a la planificación del programa. Al contrario, si uno se pregunta quiénes pueden ser estos actores comunitarios que deberán trazar esta relación de confianza con los operadores, se concluye que no puede ser cualquier persona del barrio, por el mero hecho de habitarlo. En principio tiene que manifestar una preocupación por el barrio y por los jóvenes y esta preocupación debe ir en una dirección similar a la del programa. Como veremos, en la práctica esto implica que son mujeres que realizan algún tipo de trabajo voluntario.

Si tenemos en cuenta que uno de los principales modos en los que los actores comunitarios colaboran es mediante la circulación de información y que la comunidad no constituye un agregado homogéneo de personas, es lógico concluir que no existe un único relato del habitante de la villa, sino que hay distintas perspectivas e intereses en juego. Por este motivo, los operadores intentan obtener varias versiones de los hechos pues saben que la villa no es homogénea. Así, sobre cada hecho se darán distintas versiones y finalmente decidirán a quién creerle, en la mayoría de los casos, gracias a la confianza que depositen en cada una de las partes. Así, más allá del reconocimiento de la heterogeneidad, el resultado es la construcción de un relato único que puede componerse de una o varias versiones. En este sentido, es útil considerar las reflexiones que realiza Pottier ${ }^{36}$ en el campo de la antropología del desarrollo acerca del conocimiento local: en general es tratado como si fuera homogéneo e incontestable. Siguiendo a Foucault advierte que las representaciones dominantes y externas del conocimiento local tienen más que ver con un ejercicio de poder que con realidades objetivas.

Al mismo tiempo, la preocupación por obtener información relacionada con casos de delito, implica ciertas contradicciones. En principio, aquellas personas que son vistas como preocupadas por el barrio por los operadores, pueden ser vistas como delatores por otros vecinos. Y además, los operadores requieren información para trabajar, pero desde la perspectiva burocrática muchas veces la información que manejan debería implicar la realización de denuncias y el hecho de no hacerlas podría implicar la acusación de encubrimiento. Por este motivo, en muchas ocasiones cuando recaban información, los operadores indican que quieren saber acerca del bienestar de algún beneficiario pero aclaran que no quieren saber dónde está en el caso de que esté prófugo. Este conocimiento podría implicar no sólo una acusación de encubrimiento, sino también el riesgo de ser vistos como delatores en el barrio, lo cual no sólo podría implicar una pérdida de confianza que minaría el alcance del programa, sino también un peligro para la seguridad personal.

36 Pottier, Johan, 1996 
En términos generales, puede decirse que se observa en la perspectiva del programa una cierta evaluación moral relacionada con los habitantes de la villa y sus relaciones y su predisposición a brindar conocimiento o a participar.

Así como el tema del conocimiento local ha sido abordado por la literatura del desarrollo, el vocablo de participación ha merecido extensas consideraciones. La idea de participación en los procesos de desarrollo parece ser un avance democrático, sin embargo sus usos e implicancias también deben ser problematizadas. En ocasiones ocurre que la participación se entiende como los procesos de información o consulta a los participantes: en este caso se trata de una participación superficial; en cambio, nos encontramos con lo que la literatura especializada denomina empoderamiento cuando los procesos de participación significan la transformación en los modos en que la gente negocia con las instituciones de todos los días ${ }^{37}$.

Para comprender la complejidad que reviste el concepto de participación es pertinente retomar lo planteado por Cardarelli y Rosenfeld ${ }^{38}$ : las autoras estudian los usos de la participación en América Latina en la década del 90. Señalan que en los países en los que se verifica lo que O'Donnel denominó "ciudadanías de baja intensidad", los más pobres resuelven sus necesidades de forma cada vez

37 Cornwall, Andrea, 2000, $92 \mathrm{p}$.

38 Cardarelli, Graciela y Rosenfeld, Mónica, 1998. más dependiente del Estado. Así, el Estado es el distribuidor central de bienes necesarios, de modo que construye hegemónicamente las ofertas y demandas y los procesos y alcances participativos de los programas. Esto ocurre en el contexto de una política social compensatoria subordinada a las demandas de modernización económica, lo cual implica la descentralización, focalización y privatización con transferencia de responsabilidades a la sociedad civil.

Como señalan las mismas autoras, estos modelos de participación que se despliegan en esferas localistas, a la manera de solidaridades próximas, pueden resultar altamente positivos para la población beneficiaria en términos de satisfacción de demandas y necesidades. Y además, pueden transformase en acciones colectivas con mayor capacidad de agregar intereses pese a los objetivos de control social que animan a los agentes convocantes.

En realidad, este tipo de participación comunitaria está íntimamente vinculada con los procesos de descentralización que según algunos autores conlleva una democratización, incorporando a los sectores excluidos y marginados. Sin embargo, las autoras advierten acerca del peligro de territorializar la pobreza. En efecto, este tipo de programas corre el riesgo de reforzar las tendencias a que los pobres permanezcan confinados a los límites del 
barrio o de la villa, obteniendo sus ingresos y generando sus actividades dentro del barrio.

Además, como señala De Mattos ${ }^{39}$, en las propuestas de descentralización subyace el supuesto de armonía social en las comunidades. Y en el caso específico de este programa, muchas acciones tienden a intentar recuperar una armonía que se supone que alguna vez caracterizó al barrio. Así, los operadores interpretan los conflictos entre los actores comunitarios como una falta de colaboración o como fallas o rupturas en la red.

Como mencionamos antes, Mariana organiza el trabajo de las chicas del comedor. Esta organización implica la administración de recursos: la comida (que viene del Estado) y el trabajo voluntario de las otras compañeras, el lugar del que disponen, que sirve también como sede de cursos de capacitación, un jardín de infantes o de este programa. Y este nivel de trabajo que administra Mariana no se verifica en un clima de pura armonía, sino que se dan enfrentamientos y conflictos que en ocasiones llegan a los oídos de los operadores. Cuando conocí a Mariana sus enfrentamientos más fuertes eran con Patricia hasta que esta se volvió a su provincia de origen; cuando ello ocurrió los operadores esperaban un período de armonía, pero en lugar de ello, Mariana les dijo: "ahora tengo muchas patricitas". El modo de relacionarse con estos conflictos por

39 De Mattos, Carlos, 1989. parte de los operadores consistía básicamente en escucharlos e interpretarlos como rupturas de la red, e intentar no intervenir en ellos. María Clara sostenía que había que dejar de reunirse por separado con las personas enfrentadas porque no se quería prestar al conventillo. Se esperaba que esta neutralidad mantuviera las relaciones de confianza entre los operadores y todos los actores al mismo tiempo que evitaría profundizar los conflictos existentes.

Por otra parte, intentar referirse a la participación implica abordar un término polisémico. Un análisis fructífero debe tener en cuenta quién participa y de qué manera lo hace. En este sentido, el perfil del programa se destaca fuertemente pues -más allá de los actores comunitarios que son convocados a participar en diversos ámbitos- sus beneficiarios no suelen ser considerados por el resto de los programas o proyectos de actividades barriales. En efecto, se trata preferencialmente de jóvenes con trayectorias delictivas que no suelen ser convocados por otros tipos de programas. Tal como lo remarca Cornwall ${ }^{40}$, es importante prestar atención a la autoexclusión, al hecho de que es posible elegir no participar. En este sentido, las instituciones pocas veces tienen en cuenta los costos que tiene la participación para los agentes que la practican. Y - sin embargo- también cabe preguntarse cómo participan, en calidad de qué y si su inclusión en un programa de este tipo no implica, al

40 Cornwall, Andrea, 2000. 
mismo tiempo, un etiquetamiento estigmatizante, a la vez que una el beneficio de obtener un ingreso mensual.

Por otro lado, a la hora de hablar de participación hay que tener en cuenta las desigualdades de poder que el uso de este vocablo tiende a opacar. Como señala Mosse ${ }^{41}$, las negociaciones de planificación no se hacen entre iguales. Así, el conocimiento local no corrige ni moldea proyectos, sino que se articula y es estructurado por los proyectos. Aún más, en la mayoría de los proyectos la participación constituye un valor político pero suele quedarse en un modo de hablar antes que en un modo de ser.

En este campo, se genera un tipo particular de participación. Partimos de la idea de que la misma nunca implica la efectiva igualdad entre los que desarrollan, quienes se desarrollan y los que resisten el desarrollo.

En el campo de la teoría del desarrollo, surge una preocupación posterior a la de participación: el capital social ${ }^{42}$. Uno de los principales problemas de este concepto es que implica una visión romantizada de la pobreza, igualando -en cierto sentido- al capital social con cohesión o solidaridad. Por ejemplo, Kliksberg ${ }^{43}$ recupera la definición de Putnam para señalar que el capital social es el grado de confianza existente entre los actores de una sociedad y el nivel de asociatividad. Más tarde, siguiendo a otros autores agrega que el capital social tiene un rol importante en superar las fallas del mercado mediante acciones colectivas y usos comunitarios de los recursos. Más allá de realizar afirmaciones contradictorias a lo largo del texto, sostiene que los grupos desfavorecidos tienen valores que les dan identidad. De modo que el capital social puede ser una palanca para el desarrollo. En este sentido, podemos afirmar que desde esta perspectiva, los mismos problemas de violencia que pueden generarse por la precariedad de las condiciones de vida o por la superpoblación pueden ser afrontados por la cohesión de esta población. En este punto, parece que la comunidad que es acusada por el programa de estar quebrada, debería transformarse en la llave para resolver los mismos problemas que ella genera.

Si a esta tensión presente en la idea de comunidad le sumamos el hecho de que el programa toma a ciertos actores comunitarios con los que construye una relación de confianza, es posible sostener que se trabaja con la idea de que esos actores representan la preocupación por el barrio. Sin embargo, esta representatividad es problemática y debe ser analizada. Máxime, cuando se tiene en cuenta que los actores comunitarios son mujeres. En este

41 Mosse, David, 2001.

42 Molineux, Maxine, 2002.

43 Kliksberg, Bernardo, 2000. 
sentido, Parpart ${ }^{44}$ señala que cuando una mujer opina se la toma como si fuera representativa del resto, a diferencia de los hombres.

La condición de género también merece ser problematizada cuando se considera que el trabajo que los actores comunitarios realizan es voluntario. Esto no es una característica exclusiva del programa; de hecho estos actores comunitarios realizan otros trabajos voluntarios que no están relacionados con el programa y es en función de esos trabajos comunitarios que han sido seleccionados para colaborar en la prevención del delito. A modo de ejemplo, basta con mencionar que las encargadas del comedor no perciben sueldo alguno y cocinan todos los días hábiles para más de cien personas y prestan las instalaciones para distintas actividades (apoyo escolar, el funcionamiento de un jardín de infantes, talleres diversos), teniendo que hacerse cargo de la limpieza de las instalaciones. Los problemas que este tipo de trabajos pueden ocasionar pueden comprenderse al considerar que la retribución de una bolsa de comida semanal para sus hogares que ellas mismas se adjudicaron debe permanecer oculta de la comunidad, pues suelen ser desconfiadas y acusadas de robarse la comida.

Probablemente, desde una lógica externa este trabajo se nos aparezca como extraño. ¿Por qué deberían ser las mujeres las que se encarguen de la

44 Parpart, Jane, 2002. alimentación y la seguridad del barrio? Como sostiene Sarti ${ }^{45}$, en la vida cotidiana, el barrio aparece metafóricamente como un espacio femenino. Y ello está relacionado con que el fundamento de las relaciones de género está en la noción de reciprocidad como un principio de organización social. En este sentido, cuando Molyneux ${ }^{46}$ da cuenta de que la evidencia muestra que las mujeres de bajos ingresos son las que más participan de lazos comunitarios, señala que esto tiene que ver con el supuesto de que las mujeres están más dispuestas a servir a sus familias o comunidades porque tienen menos inquietudes individuales. De modo que se naturaliza el trabajo de servicio de las mujeres, lo que redunda en que sean reclutadas para trabajo voluntario. Otro efecto de estos usos del capital social es que cuando se lo considera la panacea que puede compensarlo todo, las mujeres voluntarias se quedan con responsabilidades excesivas. Y en general las políticas le prestan poca atención a la desigualdad de género.

En el caso particular que analizamos, el hecho de que las principales colaboradoras del programa sean mujeres no es trabajado por el programa, sino que es aceptado. A ello se suma el hecho de que Mariana y otras de las chicas son bolivianas, condición poco valorada entre los jóvenes beneficiarios. De este modo, la acción del programa pone

45 Sarti, Cynthia, 1989

46 Molineux, Maxine, 2002 
a disposición de estos jóvenes el trabajo voluntario (que mantiene en condiciones el lugar de reuniones y en ocasiones se ofrecen bebidas o comida durante las reuniones) producido por personas a las que no se reconoce lo suficiente.

Por ejemplo cuando los operadores propusieron que los beneficiarios colaboraran con el comedor terminando los trabajos de pintura de las paredes y lijado de los bancos. Ni Mariana ni las chicas mostraron mucha expectativa, decían que el trabajo hacía falta pero no creían que lo fueran a terminar y los jóvenes -en su mayoría argentinos- se habían mostrado dispuestos a realizar trabajos "por el barrio". Pero cuando debía decidirse qué trabajos se realizarían comenzaron los desacuerdos. La primera propuesta de los operadores del programa había consistido en arreglar algunos pasillos de una zona cercana al comedor. La respuesta fue unánime: "eso está lleno de bolitas, que arreglen ellos". Luego de una prolongada discusión, los operadores propusieron arreglar el comedor de Mariana puesto que muchos de ellos eran beneficiarios del comedor, a la vez que por ser beneficiarios del lugar utilizan las instalaciones. Algunos aceptaron la propuesta, mientras que otros dijeron que no querían trabajar para las bolivianas y -consistentemente- se ausentaron en las siguientes reuniones.

338 revista invi № 74 / Mayo 2012 / Volumen № 27: 315-341
Entonces, el género y la nacionalidad son criterios de clasificación social que ubican a estos actores comunitarios en una posición desfavorecida dentro de las jerarquías barriales y esta posición no puede ser aceptada por el programa pero su trabajo puede evidenciar las contradicciones.

\section{Comentarios finales}

Para finalizar, nos interesa hacer algunas consideraciones relativas a posibles evaluaciones del programa y al rol que la comunidad puede jugar en las políticas de prevención del delito. Tal como señala Sozzo, estos programas están sujetos a ciertas dificultades en el contexto argentino. Para comenzar, se evidencia un problema de escala: se trabaja con grupos de destinatarios muy limitados al interior de los territorios de exclusión social. Por lo tanto, por más que se dieran cambios en las trayectorias de vida de los beneficiarios, no se verificaría un impacto en los volúmenes de microcriminalidad. En segundo lugar, se trata de un tipo de intervenciones pobremente financiadas. Por último, en el ámbito local, la ejecución se ubica en oficinas no necesariamente apropiadas.

En un nivel más general, Chinchilla y Rico ${ }^{47}$ señalan que la prevención social corre el riesgo de banalizarse. Ello implica que el resultado del proceso

47 Chinchilla, Laura y Rico, José, 2011. 
sea el financiamiento de actividades de animación sociocultural desprovistas de impacto observable en la delincuencia.

Por otro lado, resulta importante considerar las exigencias a las que están sometidos los roles adjudicados a los operadores y a los actores comunitarios. En ellas, podremos encontrar explicaciones para muchas de las dificultades en las que en ocasiones se encuentra el programa.

Si la relación que debe trazarse entre operadores y actores comunitarios es de confianza y -al mismo tiempo- de reciprocidades, ello implica demandas en ambos sentidos. Lo mismo ocurre con la relación entre operadores y beneficiarios. Ahora bien, en este entramado de relaciones siempre habrá demandas insatisfechas.

Si la producción de ciertos tipos de delitos está relacionada con privaciones y son estas relaciones que la política pública introduce las que deben resolver estas privaciones, los operadores y los actores comunitarios aparecen en última instancia como responsables de que el proceso de transformación de los jóvenes no se produzca.
Si bien el programa está sostenido por muchos procesos de reflexión, hay momentos en los que en la práctica se trabaja con ideas de participación y solidaridad que tienen cierta inocencia y que acaban responsabilizando a la comunidad de no producir las compensaciones necesarias para que los jóvenes adquieran aquello que la estructura les niega.

Ciertos clivajes que obstaculizan la profundización de los vínculos entre actores comunitarios y beneficiarios son el género y la nacionalidad. En efecto, para muchos beneficiarios profundizar sus vínculos con mujeres bolivianas constituye un proceso indeseable, puesto que son atributos poco valorados desde su perspectiva. La expresión de su disconformidad frente a demandas de los operadores suele ser tomada desde la perspectiva del programa como procesos de ruptura de la comunidad o de la red de solidaridad, cuando en realidad son inherentes al desarrollo de estos vínculo. Tal como señala Mary Douglas ${ }^{48}$, escribir sobre cooperación y solidaridad implica escribir sobre rechazo y desconfianza. El problema es que la desconfianza - que afecta a todo el mundo - en ocasiones es tan profunda que hace que la cooperación resulte imposible.

48 Douglas, Mary, 1996. 


\section{Bibliografía}

AUYERO, Javier. ¿Favores por votos? Estudios sobre clientelismo político contemporáneo. Buenos Aires, Losada. 1997. 234 p. ISBN 950-03-7179-0.

BECKER, Howard. Outsiders. Hacia una sociología de la desviación. Buenos Aires, Siglo XXI editores. 2009, 256 p. ISBN 978-987-629-083-8

BUFARINI, Mariel. Políticas sociales y personas en "situación de calle". Análisis de programas municipales en la Ciudad de Rosario. En: Congreso Argentino de Antropología Social (9², 2008, Posadas, Argentina), IX Congreso Argentino de Antropología Social: fronteras de la antropología. Posadas, Argentina, UNAM. 2008.

CARDARELLI, Graciela y ROSENFELD, Mónica. Las participaciones de la pobreza. Programas y proyectos sociales. Buenos Aires, Paidós. 1998, 168 p. ISBN 950-12-4502-0.

CHINCHILLA, Laura y RIC0, José. La prevención comunitaria del delito: perspectivas para América Latina. [En línea] Centro para la administración de justicia. Florida International University. [Fecha de consulta: 20 de septiembre 2011]. Disponible en: www2.fiu.edu/ caj/Chinchilla\%20Prevencion\%20del\%20Delito\%202.doc

CIAFARDINI, Mariano. Delito urbano en la Argentina. Las verdaderas causas y las acciones posibles. Buenos Aires, Ariel. 2005, 197 p. ISBN 950-912294-7.

340 revista invi № 74 / Mayo 2012 / Volumen № 27: 315-341
CORNWALL, Andrea. Beneficiary, consumer, citizen: perspectives of participation for poverty reduction. Gothenburg, Novum Grafiska. 2000, 92 p. ISBN 91-586-8919-2.

DE MATTOS, Carlos. La descentralización: ¿una nueva panacea para enfrentar el subdesarrollo regional? En LAURELLI, Elsa y ROFMAN, Alejandro. Descentralización del Estado: requerimientos y políticas en la crisis. Buenos Aires, Ediciones CEUR. 1989, 335 - 365 p. ISBN 950-9370-14-2.

DIRECCIÓN GENERAL DE ESTADÍSTICA Y CENSOS. Resultados provisionales del Censo Nacional de Población. Hogares y viviendas 2010 en la Ciudad de Buenos Aires [En línea] Gobierno de la Ciudad de Buenos Aires [Fecha de consulta: 22 de septiembre de 2011]. Disponible en: http://estatico.buenosaires.gov.ar/areas/hacienda/sis_estadistico/resultados_provisionales_censo_2010.pdf

DOUGLAS, Mary. Cómo piensan las instituciones. Madrid, Alianza. 1996, 202 p. ISBN84-206-2853-0.

FONSECA, Claudia. Família, fofoca e honra. Etnografia de relaçoes de gênero e violência em grupos populares. Porto Alegre, Editora da UFRGS. 2004. 245 p. ISBN85-7025-783-X.

FREDERIC, Sabina. Buenos vecinos, malos políticos. Moralidad y política en el Gran Buenos Aires. Buenos Aires, Prometeo. 2004. 284 p. ISBN $950-$ 9217-83-2.

GUTIÉRREZ, Andrea y REARTE, Julio. Segregación y accesibilidad a servicios públicos de transporte en la Ciudad de Buenos Aires. En: Brasileiro, A y 
Marques da Silva, P (eds.) Panorama Nacional da pesquisa en Transportes. Río de Janeiro, ANPET. 2006, p.829-840. ISBN 85-87893-12-82.

HERZFELD, Michael. The social production of indifference. Exploring the symbolic roots of western bureaucracy. Chicago, University of Chicago Press edition. 1993. 207 p. ISBN 0-226-32908-9.

JUHEM, Philippe. "Civiliser" la banlieue. Logiques et conditions d'efficacité des dispositifs étatiques de régulation de la violence dans les quartiers populaires. Revue française de science politique. 50 (1): 53-72, 2000. DOI 10.3406/rfsp.2000.395453.

KLIKSBERG, Bernardo. El rol del capital social y de la cultura en el proceso de desarrollo En KLIKSBERG, Bernardo y TOMASINI, Luciano. Capital social y cultura: claves estratégicas para el desarrollo. Buenos Aires, Fondo de Cultura Económica. 2000, 19 - 58 p. ISBN 978-95055 - 73684.

MANCINI, Inés. Formas de conflicto e intervención estatal en una villa de emergencia. Urvio. Revista Latinoamericana de Seguridad Ciudadana. (7): 11011, mayo de 2009. ISSN 1390-3691.

MARLOW, Alan y PITTS, John. "Remember the Alamo": action research and crime prevention in a Igh - Crime Neighborhood. International criminal justice review. 9: (69): 69-87, mayo de 1999. DOI 10.1177/105756779900900105.

MAUSS, Marcel. Ensayo sobre los dones. Razón y forma del cambio en las sociedades primitivas. En: Sociología y Antropología. Madrid, Tecnos, 1966. $263 \mathrm{p}$.
MíGUEZ, Daniel. Delito y cultura. Los códigos de la ilegalidad en la juventud marginal urbana. Buenos Aires, Biblos. 2008, 254 p. ISBN 978-950-786-696-8.

MOLINEUX, Maxine. Gender and the silences of social capital: lessons from latin America. En Development and change. 33 (2): 167 - 188, abril de 2002. DOI 10.1111/1467-7660.00246.

MOSSE, David. People's knowledge, participation and patronage: operations in rural development. En COOKE, Bill y KOTHARI, Uma. Participation: the new tyranny? Londres, Zed. 2001, 16-35 p. ISBN 1-85649-794-1.

PARPART, Jane. Lessons from the field. Rethinking empowerment, gender and development from a post (post?) development perspective. En SAUNDERS, K. Feminist post development thought. Londres, Zed books. 2002, 41-56 p. ISBN 9788189013240

POTTIER, Johan. Modern information warfare versus empirical knowledge: framing the crisis in eastern Zaire, 1996. En Pottier, J; Bicker, A; Sillitoe, P. Negotiating local knowledge. Power and identity in development. Londres, Pluto Press. 2004. p. 215-240. ISBN 0745320074.

SARTI, Cynthia. Reciprocidade e hirarquia: relaçoes de gênero na periferia de Sao Paulo. Cad. Pesq, Sao Paulo (70): 38-46, agosto 1989. ISSN 0100-1574.

SOZZO, Máximo. Gobierno local y prevención del delito en la Argentina. Urvio. Revista Latinoamericana de Seguridad Ciudadana. (6): 58-73, enero de 2009. ISSN I390-369I. 